\title{
Accurately modeling the internet topology
}

\author{
Shi Zhou* and Raúl J. Mondragón ${ }^{\dagger}$ \\ Department of Electronic Engineering, Queen Mary College, University of London, London E1 4NS, United Kingdom
}

(Received 4 February 2004; revised manuscript received 21 July 2004; published 3 December 2004)

\begin{abstract}
Based on measurements of the internet topology data, we found that there are two mechanisms which are necessary for the correct modeling of the internet topology at the autonomous systems (AS) level: the interactive growth of new nodes and new internal links, and a nonlinear preferential attachment, where the preference probability is described by a positive-feedback mechanism. Based on the above mechanisms, we introduce the positive-feedback preference (PFP) model which accurately reproduces many topological properties of the AS-level internet, including degree distribution, rich-club connectivity, the maximum degree, shortest path length, short cycles, disassortative mixing, and betweenness centrality. The PFP model is a phenomenological model which provides an insight into the evolutionary dynamics of real complex networks.
\end{abstract}

DOI: 10.1103/PhysRevE.70.066108

PACS number(s): 89.75.-k, 87.23.Ge, 05.70.Ln

\section{INTRODUCTION}

Recently there has been a considerable effort to understand the topology of complex networks [1-6]. Of particular interest are complex networks obtained from evolving mechanisms, like the internet or the worldwide web, as they are so influential in our daily life. The degree $k$ of a node is the number of links which have the node as an end point, or equivalently the number of nearest neighbors of the node. The statistical distribution of the degree $P(k)$ gives important information of the global properties of a network and can be used to characterize different network topologies. The internet has been studied in detail [7-13] since the measured data [14-17] became available. Now, it is well known that the internet can be represented as a scale-free network, where the degree distribution is a power law $P(k) \sim k^{-\gamma}$. The exponent $\gamma$ of the internet at the autonomous systems (AS) level is approximately 2.22 (see Figs. 1 and 2).

Barabási and Albert (BA) [19] showed that it is possible to grow a network with a power-law degree distribution by using a preferential-growth mechanism: starting with a small random network, the system grows by attaching a new node with $m$ links to $m$ different "old" nodes that are already present in the system ( $m=3$ to obtain internetlike networks); the attachment is preferential because the probability that a new node will connect to node $i$, with degree $k_{i}$, is

$$
\Pi(i)=\frac{k_{i}}{\sum_{j} k_{j}} .
$$

\footnotetext{
*Also at Department of Electronic \& Electrical Engineering, University College London, Adastral Park Campus, Ross Building, Martlesham Heath, Ipswich IP5 3RE, United Kingdom; email address: s.zhou@adastral.ucl.ac.uk

${ }^{\dagger}$ Email address: r.j.mondragon@elec.qmul.ac.uk
}

The BA model generates networks with the power-law exponent $\gamma=3[20]$.

Based on the BA model, a number of evolving network models $[2,4,6,13]$ have been introduced to obtain degree distributions with other power-law exponents. Some of these new models have been used to model the internet. However, a network model based solely on the reproduction of the power-law exponent of the degree distribution has its limitations, as it will not describe the internet hierarchical structure [8]. In the next section we investigate two properties of the internet that were not accurately modeled by the existing models, namely, the rich-club connectivity [21] among highdegree nodes and the maximum degree of the network. The accurate modeling of these two properties was our motivation for developing a network model. In Sec. III we introduce the positive-feedback preferential (PFP) model, which is a phenomenological model of the AS-level internet topology. Section IV presents the validation of the model and in Sec. V are the conclusions of this work.

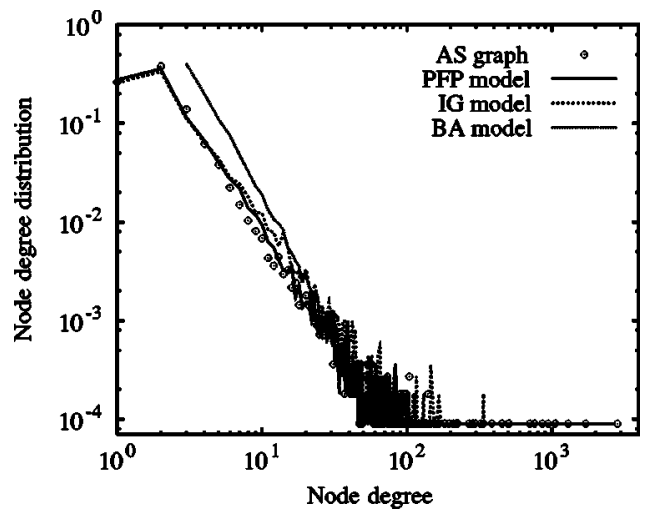

FIG. 1. Degree distribution. The AS-level internet topology data set used in this research is a trace-route-derived AS graph measured in April 2002 [18]. The models illustrated are the positive-feedback preferential (PFP), interactive growth (IG), and Barabási-Albert (BA) models. 


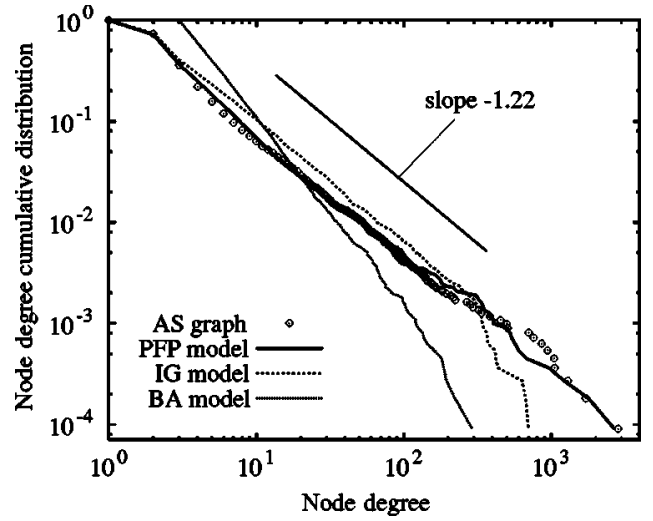

FIG. 2. The cumulative degree distribution $P_{c m m}(k)$ of the AS graph decays as $P_{c m m}(k) \sim k^{-1.22}$; hence the degree distribution $P(k) \sim k^{-\gamma}$ with exponent $\gamma \simeq 2.22$ [7].

\section{CHALLENGES IN ACCURATE MODELING OF THE INTERNET}

\section{A. The rich club}

Scale-free networks can be grouped into assortative, disassortative, and neutral networks [22-24]. Social networks (e.g., the coauthorship network) are assortative networks, in which high-degree nodes prefer to attach to other highdegree nodes. Information networks (e.g., the worldwide web and the internet) and biological networks (e.g., protein interaction networks) have been classified as disassortative networks, in which high-degree nodes tend to connect with low-degree ones.

While the AS-level internet is disassortative [10,11], this property does not imply that the high-degree nodes are tightly interconnected to each other (see Fig. 3). One of the structural properties of the AS-level internet is that it contains a small number of high-degree nodes. We call these nodes "rich" nodes, and the set containing them the "rich club." The inter-connectivity among the club members is quantified by the rich-club connectivity [21] which is defined as follows. The rank $r$ of a node denotes its position on a list of all nodes sorted in decreasing degree. If the network has $N$ nodes then $r \in[1, N]$. If the rich club consists of the first $r$ nodes in the rank list, then the rich-club connectivity $\phi(r / N)$ is defined as the ratio of the number of links connecting the club members over the maximum number of allowable links, $r(r-1) / 2$. The rich-club connectivity measures how well club members "know" each other. A rich-club connectivity of 1 means that all the members have a direct link to any other

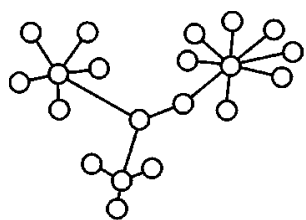

(a)

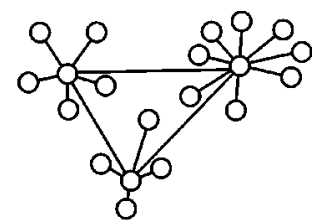

(b)
FIG. 3. Two disassortative networks. (a) High-degree nodes are loosely interconnected. (b) High-degree nodes are tightly interconnected.

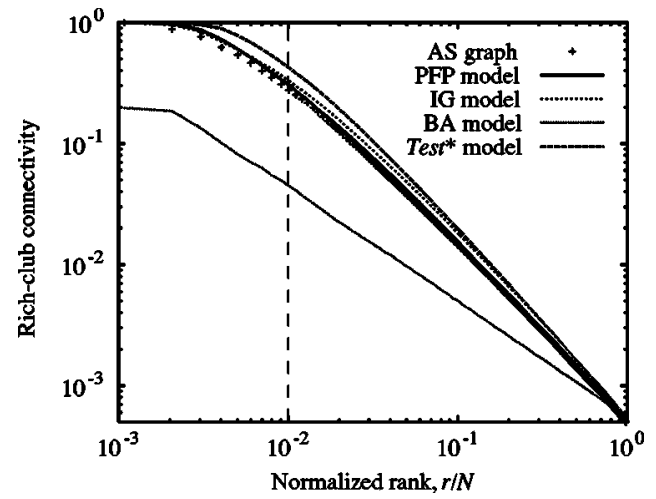

FIG. 4. Rich-club connectivity $\phi(r / N)$ vs normalized rank $r / N$. The top $1 \%$ best-connected nodes are marked with the vertical hash line.

member, i.e., they form a fully connected subgraph.

Figure 4 shows the rich-club connectivity as a function of the rank normalized by the number of nodes. It is clear that in the AS graph the high-degree nodes are tightly interconnected. The top $1 \%$ best-connected nodes of the AS graph have $27 \%$ of the possible interconnections, compared with only $4.5 \%$ obtained from a network topology generated using the BA model which has the same number of nodes as, and slightly larger number of links, than the AS graph (see Table I).

The rich club consists of highly connected nodes, which are well interconnected between each other, and the average hop distance among the club members is very small (one to two hops). The rich club is a "super" traffic hub of the network and the disassortative mixing property ensures that peripheral nodes are always near the hub. These two structural properties together contribute to the routing efficiency of the network. An internet model that does not reproduce the properties of the rich club will underestimate the actual network's routing efficiency (shortest path length) and routing flexibility (alternative reachable paths), and also it will overestimate the network robustness under node attack [25].

\section{The interactive growth model}

The BA model is based solely on the attachment of new nodes. However, the appearance of new internal links among old nodes has also been observed in the evolution of the internet $[10,11]$. During the last few years, researchers have proposed a number of internet models using the appearance of new internal links, such as Dorogovtsev and Mendes' model [26], Bu and Towsley's generalized linear preference model [27], Bianconi et al.'s generalized network growth model [28], Caldarelli et al.'s model [29], and the interactive growth (IG) model [30]. In addition to the appearance of new internal links, these models have also used different preference schemes to capture selected properties of the internet.

Here we reconsider the interactive growth model as it is the precursor of the positive-feedback preference model and the IG model provides a possible way to reproduce both the power-law degree distribution and the rich-club connectivity of the AS graph. The IG model generates a network using interactive growth, where new internal links start from the 
TABLE I. Network parameters.

\begin{tabular}{cccccc}
\hline \hline & & AS graph & PFP model & IG model & BA model \\
\hline Number of nodes & $N$ & 11122 & 11122 & 11122 & 11122 \\
Number of links & $L$ & 30054 & 30151 & 33349 & 33349 \\
Average degree & $\langle k\rangle$ & 5.4 & 5.4 & 6.0 & 6.0 \\
Exponent of power law & $\gamma$ & 2.22 & 2.22 & 2.22 & 3 \\
Rich-club connectivity & $\phi(r / N=0.01)$ & 0.27 & 0.30 & 0.32 & 0.045 \\
Maximum degree & $k_{\max }$ & 2839 & 2785 & 700 & 292 \\
Degree distribution & $P(k=1)$ & $26 \%$ & $28 \%$ & $26 \%$ & $0 \%$ \\
Degree distribution & $P(k=2)$ & $38 \%$ & $36 \%$ & $34 \%$ & $0 \%$ \\
Degree distribution & $P(k=3)$ & $14 \%$ & $12 \%$ & $11 \%$ & $40 \%$ \\
Characteristic path length & $l^{*}$ & 3.13 & 3.14 & 3.6 & 4.3 \\
Average triangle coefficient & $\left\langle k_{t}\right\rangle$ & 12.7 & 12 & 10.4 & 0.1 \\
Maximum triangle coefficient & $k_{t} \max$ & 7482 & 8611 & 4123 & 64 \\
Average quadrangle coefficient & $\left\langle k_{q}\right\rangle$ & 277 & 247 & 105.4 & 1.3 \\
Maximum quadrangle coefficient & $k_{q} \max$ & 9648 & 9431 & 8780 & 527 \\
Average $k_{n n}$ & $\left\langle k_{n n}\right\rangle$ & 660 & 482 & 103 & 20 \\
Average betweenness & $\left\langle\mathcal{C}_{B}^{*}\right\rangle$ & 4.13 & 4.14 & 4.6 & 5.3 \\
Maximum betweenness & $\mathcal{C}_{B}^{*} \max$ & 3237 & 3419 & 1002 & 1064 \\
\hline \hline
\end{tabular}

host nodes, which are the old nodes that new nodes are attached to. The IG model starts with a small random network. At each time step, (1) with probability $p \in(0,1)$, a new node is attached to one host node and two new internal links appear between the host node and two other old nodes (peer nodes); (2) with probability $1-p$, a new node is attached to two host nodes and one new internal link appears between one of the host nodes and a peer node.

In the actual internet, new nodes bring new traffic load to its host nodes. This results in both the increase of traffic volume and the change of traffic pattern around host nodes and triggers the addition of new links connecting host nodes to peer nodes in order to balance network traffic and optimize network performance. From numerical simulations, we found that when $p=0.4$ the interactive growth model also satisfies the following two characteristics observed [9-12] in the internet measurements. First, the majority of new nodes are added to the system by attaching them to one or two old nodes $(m \leqslant 2)$. Second, the degree distribution of the AS graph is not a strict power law as it has more nodes with degree 2 than nodes with degree $1[P(2)=38 \%>P(1)$ $=26 \%$; see Table I]. The IG model uses the BA model's linear preference of Eq. (1) in the attachment of new nodes and the appearance of new internal links. As shown in Fig. 1, Fig. 2, Fig. 4, and Table I, the IG model closely resembles both the power-law degree distribution and the rich-club connectivity of the AS graph.

\section{B. Maximum degree}

The IG model still has its limitations. The maximum node degree $k_{\max }$ present in the AS graph is nearly a quarter of the number of nodes $\left(k_{\max } \simeq N / 4\right)$ and is significantly larger than the maximum degree obtained by the IG and BA models using linear preferential attachment (see Table I). To over- come this shortfall, it is possible to favor high-degree nodes by using the nonlinear preferential probability [26,31]

$$
\Pi(i)=\frac{k_{i}^{\alpha}}{\sum_{j} k_{j}^{\alpha}}, \quad \alpha>1 .
$$

To examine the above nonlinear preference, here we study a so-called Test* model, which is a modification of the IG model. The Test* model uses the same interactive growth mechanism as the IG model, but it does not use the linear preference given by Eq. (1); instead it uses the nonlinear preference given by Eq. (2). Numerical experiments showed that, when $\alpha=1.15 \pm 0.01$, the Test* model generates networks with the maximum degree similar to the AS graph. However, as shown in Fig. 4, the rich-club connectivity produced by the Test* model deviates from the AS graph. For example, the $1 \%$ best-connected nodes of the Test* model have $42 \%$ allowable interconnections compared with $27 \%$ of the AS graph.

\section{POSITIVE-FEEDBACK PREFERENCE MODEL}

Based on the internet-history data, Pastor-Satorras et al. [10] and Vázquez et al. [11] measured that the probability that a new node links with a low-degree old node follows the linear preferential attachment given by Eq. (1), whereas Chen et al. [9] reported that high-degree nodes have a stronger ability of acquiring new links than predicted by Eq. (1). The internet-history data also show that at early times, the node degree increases very slowly; later on, the degree grows more and more rapidly. Taking into account these observations, we modified the IG model by using the nonlinear preferential attachment 


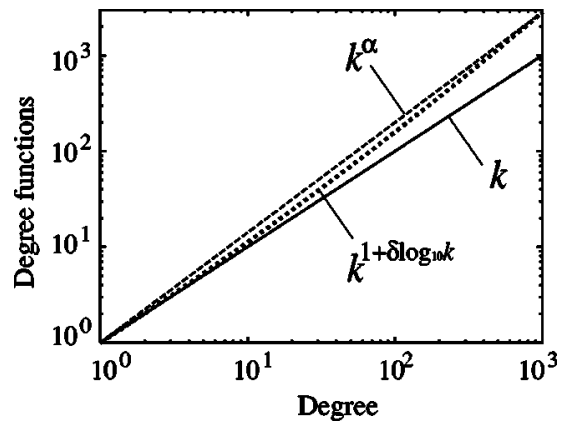

FIG. 5. Three degree functions: $k, k^{\alpha}$ with $\alpha=1.15$, and $k^{1+\delta \log _{10} k}$ with $\delta=0.048$.

$$
\Pi(i)=\frac{k_{i}^{1+\delta \log _{10} k_{i}}}{\sum_{j} k_{j}^{1+\delta \log _{10} k_{j}}}, \quad \delta \in[0,1] .
$$

We call this the positive-feedback preference model. From numerical simulations, we found that $\delta=0.048$ produces the best result. (It is interesting to notice that for $\delta=0.048$ and the maximum degree $k_{\max }=2839$ as measured on the AS graph, the exponent function of $1+\delta \log _{10} k_{\max } \simeq 1.166$, which is close to the value of $\alpha$ used in the Test* model).

We also refine the interactive growth mechanism. The PFP model starts with a small random network. At each time step, (1) with probability $p \in[0,1]$, a new node is attached to one host node, and at the same time one new internal link appears between the host node and a peer node; (2) with probability $q \in[0,1-p]$, a new node is attached to one host node, and at the same time two new internal links appear between the host node and two peer nodes; (3) with probability $1-p-q$, a new node is attached to two host nodes, and at the same time one new internal link appears between one of the host nodes and one peer node.

When $p=0.3$ and $q=0.1$, the generated PFP network has the same ratio of nodes to links as in the AS graph (see Table I). Equation (3) is used in choosing host nodes and peer nodes.

The PFP model satisfies the observations of PastorSartorras et al., Vázquez et al., and Chen et al. For lowdegree nodes, the preferential attachment is approximated by Eq. (1). For high-degree nodes, the preferential attachment increases as a nonlinear function of the node degree (see Fig. 5 ). Hence, as the time passes by, the rate of degree growth in

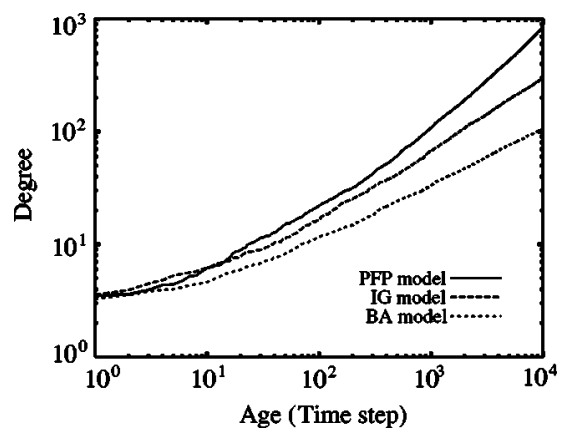

FIG. 6. Degree growth of a node.

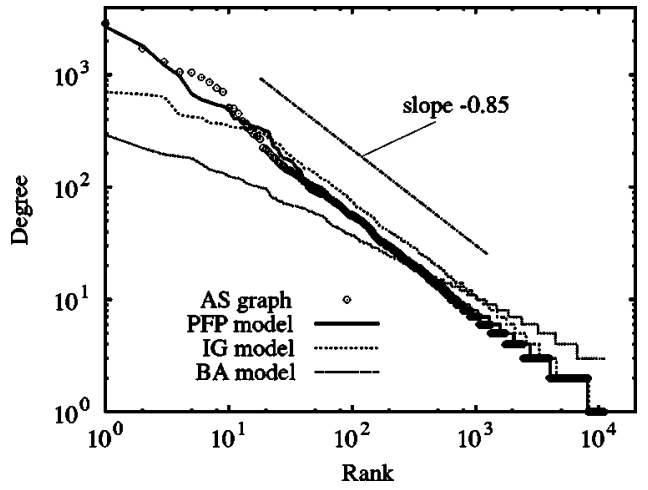

FIG. 7. Node degree $k$ vs rank $r$.

the PFP model is faster than in the IG model and the BA model (see Fig. 6).

\section{MODEL VALIDATION}

The validation was done by comparing the AS graph [18] with networks generated by the PFP model, the IG model, and the BA model. For each model, ten different networks were generated and averaged. The networks had the same number of nodes and similar numbers of links as the AS graph (see Table I).

\section{A. Degree distribution, rich-club connectivity, and maximum degree}

The PFP model produces networks that closely match the degree distribution (see Fig. 1 and Fig. 2), the rich-club connectivity (see Fig. 4), and the maximum degree (see Table I) of the AS graph. Also the networks generated using the PFP model have the same power-law relationship between degree and rank, $k \sim r^{-0.85}$, as the AS graph (see Fig. 7). In certain respects the accuracy of the PFP model to reproduce these properties is not a surprise. After all, the model was designed to match these properties.

\section{B. Shortest path length}

The average shortest path length $l$, of a node is defined as the average of the shortest-paths from the node to all other

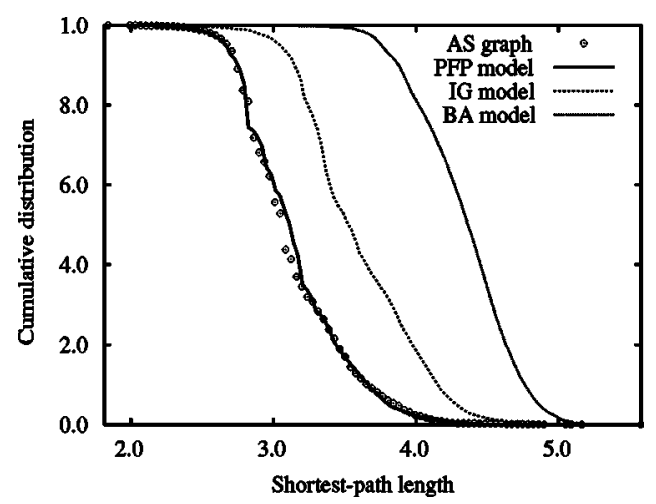

FIG. 8. Cumulative distribution of average shortest path length. 


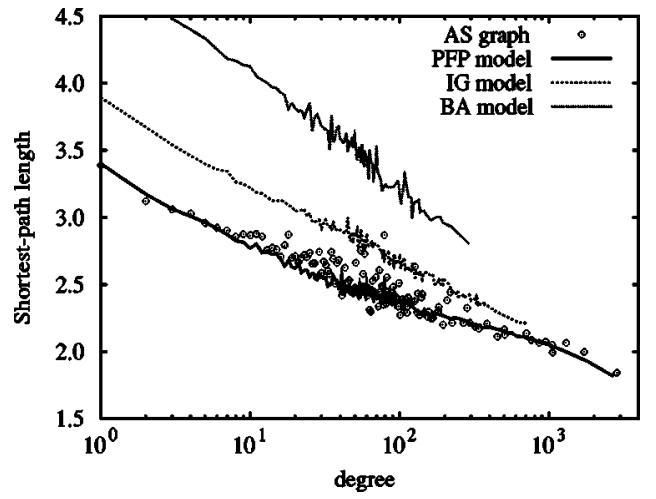

FIG. 9. Correlation between average shortest path length $l$ and degree, where $l$ is the average over nodes with the same degree.

nodes in the network. Figures 8 and 9 show that the PFP model reproduces the cumulative distribution of average shortest path length and the correlation between average shortest path length and degree of the AS graph.

The characteristic path length $l^{*}$ of a network is the average of the shortest paths over all pairs of nodes. The characteristic path length indicates the network overall routing efficiency. The AS graph is a small-world network [32] because the characteristic path length is very small compared with the network size. Table I shows that the AS graph and the networks obtained from the PFP model have nearly the same characteristic path length.

\section{Short cycles}

Cycles [28,33] encode the redundant information in the network structure. The number of short cycles (triangles and quadrangles) is a relevant property because the multiplicity of paths between any two nodes increases with the density of short cycles (note that an alternative path between two nodes can be longer than their shortest path). The triangle coefficient $k_{t}$ is defined as the number of triangles that a node shares. Similarly, the quadrangle coefficient $k_{q}$ is the number of quadrangles that a node has.

Table I shows the AS graph and the networks generated using the PFP model have higher densities of short cycles $\left(\left\langle k_{t}\right\rangle\right.$ and $\left.\left\langle k_{q}\right\rangle\right)$ than networks generated using the IG model and the BA model. Figures 10 and 11 show that the AS graph

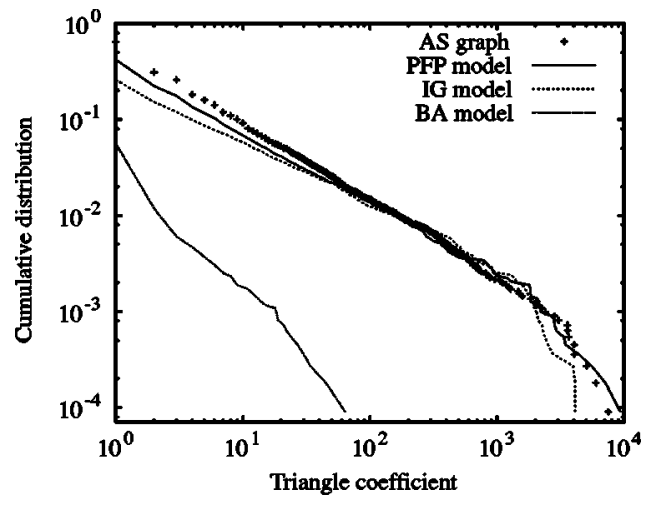

FIG. 10. Cumulative distribution of triangle coefficient.

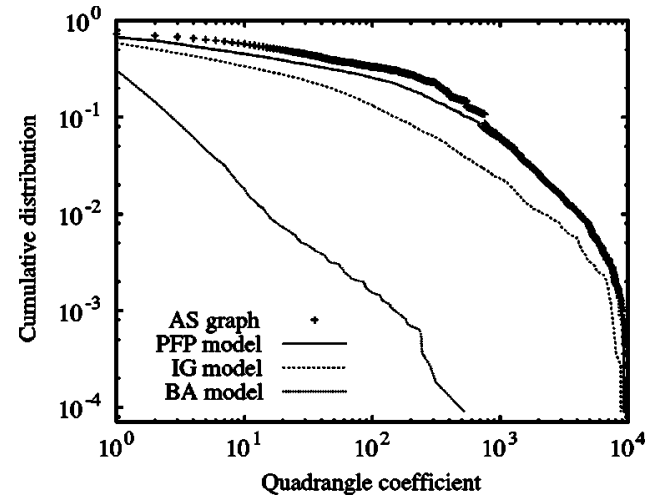

FIG. 11. Cumulative distribution of quadrangle coefficient.

and the networks obtained from the PFP model have similar cumulative distributions of short cycles. Figures 12 and 13 show that the PFP networks exhibit similar correlations between short cycles and degree as in the AS graph.

Notice that the clustering coefficient $c$ of a node can be expressed as a function of the node's degree $k$ and triangle coefficient $k_{t}$,

$$
c=\frac{k_{t}}{k(k-1) / 2} .
$$

The reason we study short cycles instead of clustering coefficients is that short cycles have the advantage of providing neighbor clustering information of nodes with different degrees.

\section{Disassortative mixing}

The internet exhibits disassortative mixing behavior $[10,11,23,24]$, where on average, high-degree nodes tend to connect to peripheral nodes with low degrees. A network's mixing pattern is identified by the conditional probability $p_{c}\left(k^{\prime} \mid k\right)$ that a link connects a node with degree $k$ to a node with degree $k^{\prime}$. This conditional probability can be indicated $[10,11]$ by $k_{n n}$, the nearest-neighbor average degree of a node with degree $k$.

Figure 14 and Table I show that on average the nearestneighbor average degree of a node in the AS graph and the

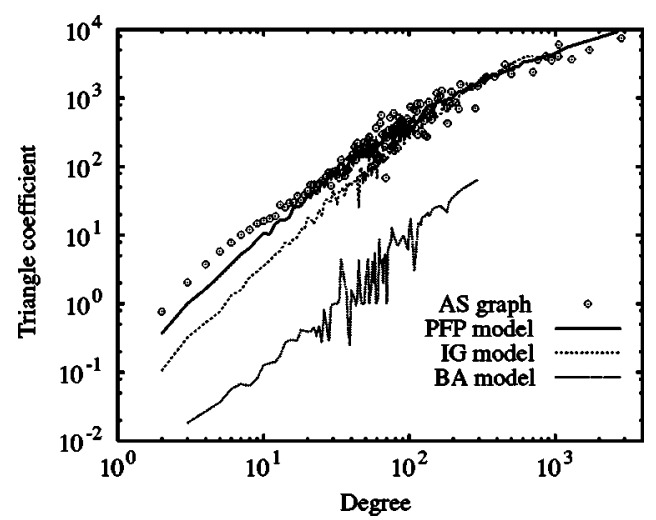

FIG. 12. Correlation between triangle coefficient $k_{t}$ and degree, where $k_{t}$ is the average over nodes with the same degree. 


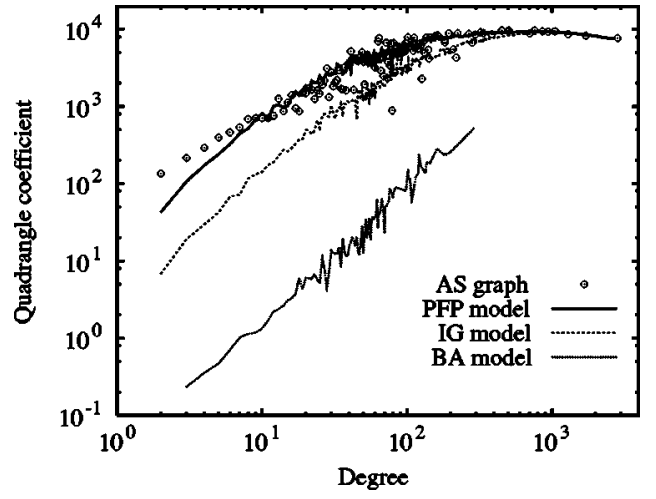

FIG. 13. Correlation between quadrangle coefficient $k_{q}$ and degree, where $k_{q}$ is the average over nodes with the same degree.

PFP networks is significantly larger than that in the IG and BA networks. Figure 15 shows that the PFP model closely reproduces the negative correlation between nearestneighbor average degree and node degree of the AS graph and therefore exhibits similar disassortative mixing as the AS graph.

\section{E. Betweenness centrality}

On a network, there are nodes that are more prominent because they are highly used when transferring information. A way to measure this "importance" is by using the concept of node betweenness centrality which is defined as follows. Given a source node $s$ and a destination node $d$, the number of different shortest paths from $s$ to $d$ is $g(s, d)$. The number of shortest paths that contain the node $w$ is $g(w ; s, d)$. The proportion of shortest paths, from $s$ to $d$, which contain node $w$ is $p_{s, d}(w)=g(w ; s, d) / g(s, d)$. The betweenness centrality of node $w$ is defined as $[34,35]$

$$
\mathcal{C}_{B}(w)=\sum_{s} \sum_{d \neq s} p_{s, d}(w),
$$

where the sum is over all possible pairs of nodes with $s \neq d$. The betweenness centrality measures the proportion of shortest paths that visit a certain node. If all pairs of nodes of a network communicate at the same rate, the betweenness cen-

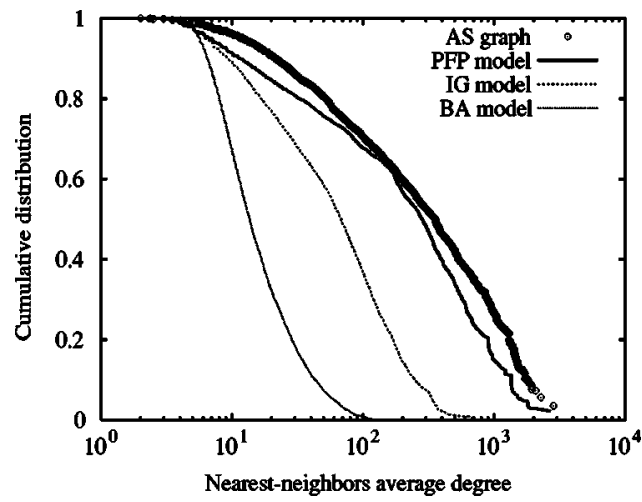

FIG. 14. Cumulative distribution of nearest-neighbor average degree.

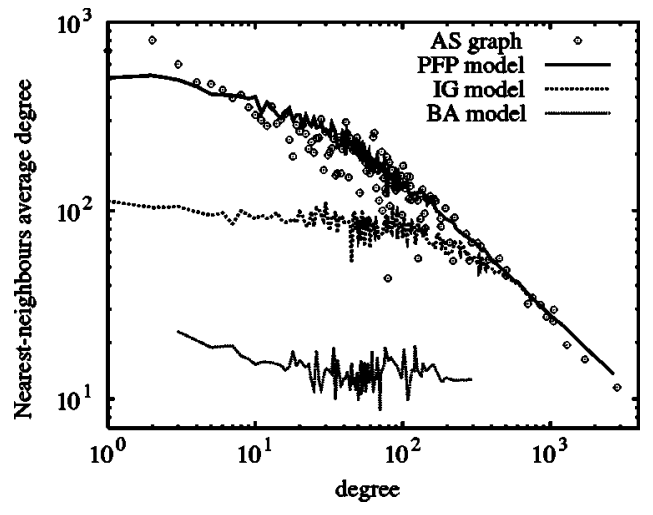

FIG. 15. Correlations between nearest-neighbor average degree $k_{n n}$ and degree, where $k_{n n}$ is the average over nodes with the same degree.

trality estimates the node's capacity needed for a free-flow status [34]. A node with a large $\mathcal{C}_{B}$ is "important" because it carries a large traffic load. If this node fails or gets congested, the consequences to the network traffic can be drastic [35]. Here the betweenness centrality is normalized by the number of nodes and denoted as $\mathcal{C}_{B}^{*}$. The average of the (normalized) betweenness centrality in a network $\left\langle\mathcal{C}_{B}^{*}\right\rangle=l^{*}+1$ [35], where $l^{*}$ is the network's characteristic path length.

Figure 16 shows that the cumulative distribution of betweenness centrality $P_{\text {cum }}\left(\mathcal{C}_{B}^{*}\right)$ of the networks exhibit similar power-law behaviors characterized by slope -1.1 ; hence $P\left(\mathcal{C}_{B}^{*}\right) \sim\left(\mathcal{C}_{B}^{*}\right)^{-2.1}[10,11]$. However, as shown in Table I, the maximum values of the betweenness centrality $\mathcal{C}_{B \text { max }}^{*}$ for the AS graph and the PFP model are significantly larger than those for the IG model and the BA model. Figure 17 shows that only the PFP model closely matches the correlation between betweenness centrality and degree of the AS graph.

\section{CONCLUSIONS AND DISCUSSION}

In summary, the PFP model accurately reproduces many of the topological properties measured in the internet at the AS level. The model is based on two growth mechanisms which are the nonlinear positive-feedback preferential attachment combined with the interactive growth of new nodes and

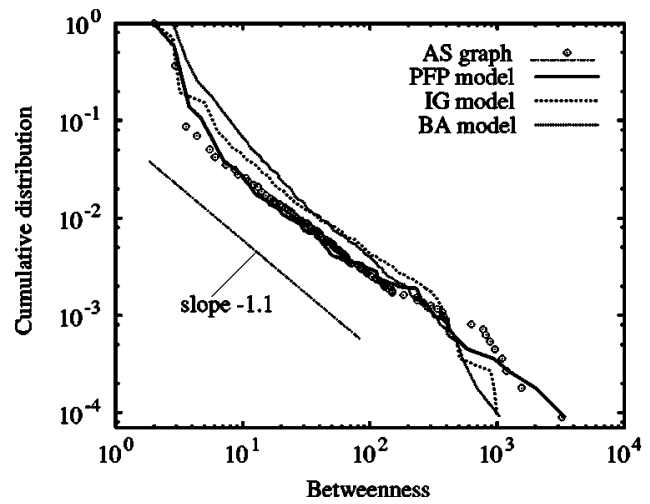

FIG. 16. Cumulative distribution of betweenness centrality, $P_{\text {cum }}\left(\mathcal{C}_{B}^{*}\right)$. 


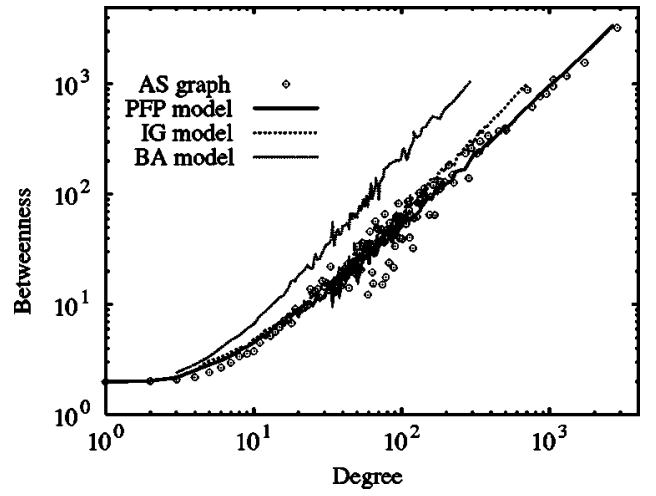

FIG. 17. Correlations between betweenness centrality $\mathcal{C}_{B}^{*}$ and degree, where $\mathcal{C}_{B}^{*}$ is the average over nodes with the same degree.

new internal links. Both the mechanisms are based on (and supported by) the observations on the internet-history data.

The positive-feedback preference means that, as a node acquires new links, the node's relative advantage when competing for more links increases as a nonlinear feedback loop. This implies that the inequality in the link-acquiring ability between rich nodes and nonrich nodes increases as the net- work evolves. Rich nodes not only become richer, they become disproportionately richer. While our initial motivation was to create a model that can accurately reproduce the richclub connectivity and the maximum degree of the AS graph, the PFP model actually captures other properties as well. Further studies are needed to explain why the internet growth seems to follow the nonlinear preferential attachment given by the PFP model and what are the consequences of this growth mechanism for the future of the internet. This research provides an insight into the basic mechanisms that could be responsible for the evolving topology of complex networks.

Finally, the validation of the model was not conducted with measurement data based on the Border Gateway Protocol tables, but with the trace-route-derived AS graph, which is regarded as a more realistic and reliable measurement of the internet [36].

\section{ACKNOWLEDGMENT}

This work was funded by the U.K. Engineering and Physical Sciences Research Council (EPSRC) under Grant No. GR-R30136-01.
[1] S. H. Strogatz, Nature (London) 410, 268 (2001).

[2] P. L. Krapivsky and S. Redner, Phys. Rev. E 63, 066123 (2001).

[3] A. L. Barabási, Linked: The New Science of Networks (Perseus, Cambridge, MA, 2002).

[4] R. Albert and A. L. Barabási, Rev. Mod. Phys. 74, 47 (2002).

[5] S. Bornholdt and H. G. Schuster, Handbook of Graphs and Networks-From the Genome to the Internet (Wiley-VCH, Weinheim, Germany, 2002).

[6] S. N. Dorogovtsev and J. F. F. Mendes, Evolution of Networks-From Biological Nets to the Internet and WWW (Oxford University Press, Oxford, 2003).

[7] M. Faloutsos, P. Faloutsos, and C. Faloutsos, Comput. Commun. Rev. 29, 251 (1999).

[8] L. Subramanian, S. Agarwal, J. Rexford, and R. H. Katz, in Proceedings of IEEE INFOCOM 2002 (IEEE Computer Society, Washington, D.C., 2002), pp. 618-627.

[9] Q. Chen, H. Chang, R. Govindan, S. Jamin, S. J. Shenker, and W. Willinger, in Proceedings of IEEE INFOCOM 2002 (Ref. [8], pp. 608-617.

[10] R. Pastor-Satorras, A. Vázquez, and A. Vespignani, Phys. Rev. Lett. 87, 258701 (2001).

[11] A. Vázquez, R. Pastor-Satorras, and A. Vespignani, Phys. Rev. E 65, 066130 (2002).

[12] S. T. Park, A. Khrabrov, D. M. Pennock, S. Lawrence, C. L. Giles, and L. H. Ungar, in Proceedings of IEEE INFOCOM 2003 (IEEE Computer Society, Washington, D. C., 2003), Vol. 3, pp. 2144-2154.

[13] R. Pastor-Satorras and A. Vespignani, Evolution and Structure of the Internet-A Statistical Physics Approach (Cambridge University Press, Cambridge, U.K., 2004).
[14] NLANR, National Laboratory for Applied Network Research, http://moat.nlanr.net/

[15] CAIDA, Cooperative Association For Internet Data Analysis, http://www.caida.org/

[16] Route Views Project, University of Oregon, Eugene, http:// www.routeviews.org/

[17] Topology Project, University of Michigan, Ann Arbor, http:// topology.eecs.umich.edu/

[18] The Data Kit \#0204 was collected as part of CAIDA's Skitter initiative. Support for Skitter was provided by DARPA, NSF, and CAIDA membership.

[19] A. L. Barabási and R. Albert, Science 286, 509 (1999).

[20] A. L. Barabási, R. Albert, and H. Jeong, Physica A 272, 173 (1999).

[21] S. Zhou and R. J. Mondragón, IEEE Commun. Lett. 8, 180 (2004).

[22] M. E. J. Newman, Phys. Rev. Lett. 89, 208701 (2002).

[23] M. E. J. Newman, Phys. Rev. E 67, 026126 (2003).

[24] S. Maslov, K. Sneppen, and A. Zaliznyak, Physica A 333, 529 (2004).

[25] S. Zhou and R. J. Mondragón, Electron. Lett. 40, 151 (2004).

[26] S. N. Dorogovtsev and J. F. F. Mendes, Europhys. Lett. 52, 33 (2000).

[27] T. Bu and D. Towsley, in Proceedings of IEEE INFOCOM 2002 (Ref. [8]), p. 638.

[28] G. Bianconi, G. Caldarelli, and A. Capocci, e-print cond-mat/ 0310339.

[29] G. Caldarelli, P. D. L. Rios, and L. Pietronero, e-print condmat/0307610.

[30] S. Zhou and R. J. Mondragón, in Proceedings of the 18th International Teletraffic Congress (ITC18), edited by J. Charzinski, Teletraffic Science and Engineering, Vol. 5a (Elsevier, 
Berlin, 2003), pp. 121-130.

[31] P. L. Krapivsky, S. Redner, and F. Leyvraz, Phys. Rev. Lett. 85, 4629 (2000).

[32] J. Watts, Small Worlds: The Dynamics of Networks between Order and Randomness (Princeton University Press, Princeton, NJ, 1999).

[33] G. Bianconi and A. Capocci, Phys. Rev. Lett. 90, 078701
(2003).

[34] K. I. Goh, B. Kahng, and D. Kim, Phys. Rev. Lett. 87, 278701 (2001).

[35] P. Holme and B. J. Kim, Phys. Rev. E 65, 066109 (2002).

[36] Y. Hyun, A. Broido, and K. Claffy, http://www.caida.org/ outreach/papers/2003/ASP/ 